\title{
Spatial-chirp compensation in dynamical holograms reconstructed with ultrafast lasers
}

\author{
Lluís Martínez-León, ${ }^{1}$ Pere Clemente, ${ }^{2}$ Enrique Tajahuerce, ${ }^{1}$ Gladys Mínguez-Vega, ${ }^{1, a)}$ \\ Omel Mendoza-Yero, ${ }^{1}$ Mercedes Fernández-Alonso, ${ }^{1}$ Jesús Lancis, ${ }^{1}$ Vicent Climent, ${ }^{1}$ \\ and Pedro Andrés ${ }^{3}$ \\ ${ }^{1}$ GROC.UJI, Department of Physics, Universitat Jaume I, E12080 Castelló, Spain \\ ${ }^{2}$ Servei Central d'Instrumentació Científica, Universitat Jaume I, E12080 Castelló, Spain \\ ${ }^{3}$ Departament d'Óptica, Universitat de València, 46100 Burjassot, Spain
}

(Received 4 November 2008; accepted 11 December 2008; published online 6 January 2009)

\begin{abstract}
A computer generated hologram $(\mathrm{CGH})$ reconstructed with a sub-100-fs laser pulse at the focal plane of a conventional refractive lens experiences a large amount of spatial chirp. We report the shaping of a 12 fs laser pulsed beam by means of a Fourier CGH implemented onto a spatial light modulator, using a hybrid diffractive-refractive lens triplet that provides spatial-chirp compensation. Experimental results demonstrate that parallel, arbitrary, and high-resolution patterning is possible with the proposed device. (C) 2009 American Institute of Physics. [DOI: 10.1063/1.3063047]
\end{abstract}

The creation of a desired diffraction pattern by employing a femtosecond laser pulse source is a matter of great interest in several hot topics of research such as high-speed microprocessing ${ }^{1}$ and multiphoton microscopy ${ }^{2}$ optical trapping of particules, ${ }^{3}$ and generation of optical vortices. ${ }^{4} \mathrm{Com}-$ puter generated holograms (CGHs) are photonic components designed to shape an optical beam into a user-defined intensity distribution in one shot. Usually, CGHs are implemented onto voltage-addressed flat displays working as phase-only spatial light modulators (SLM). This fact allows dynamic applications if the intensity pattern delivered onto the target must be changed.

It is worth remarking that when a beam passes through a $\mathrm{CGH}$ it experiences angular dispersion due to its diffractive nature. Then, after further propagation, the frequency components naturally separate in space, resulting in spatial chirp (SC). Consequently, CGHs enable precise spatiotemporal control of the femtosecond pulsed beam only in the longpulse duration regime (above $100 \mathrm{fs}$ ), as reported in the pioneer paper by Nolte. ${ }^{5}$ For shorter pulse durations, the significant bandwidth of ultrashort pulses results in a coupling of the spatiotemporal effects causing the degradation of the output image and the stretching of the pulse duration. Thus, dispersion compensation techniques are required to minimize these effects at the target.

In this letter, we experimentally demonstrate shaping of pulsed beams in the short-pulse duration regime by means of a Fourier-coded CGH implemented onto a parallel aligned nematic liquid crystal SLM (PAL-SLM) with a refreshing rate in the hertz range. High resolution is achieved through the correction of the first-order diffraction-induced SC, with a simple dispersion-reversed optical device based on Refs. 6 and 7, which corrects the angular dispersion introduced by the CGH. Due to the close relation between the angular dispersion and the pulse-front tilt, ${ }^{8}$ the proposed system also corrects, in a first-order approximation, the temporal pulse stretching caused by local reduction in the output spectrum.

Figure 1 shows the proposed setup, which basically consists of a femtosecond laser system, a phase modulator, and

${ }^{a)}$ Electronic mail: gminguez@uji.es. the SC compensation device. The femtosecond laser system is a mode-locked Ti:sapphire laser (Femtosource, Femtolaser), generating pulses with a peak wavelength of $800 \mathrm{~nm}$, a temporal width of $\sim 12 \mathrm{fs}$, and spectral width of $\sim 90 \mathrm{~nm}$ at full width half maximum. The pulse energy is adjusted with a set of neutral density filters. After passing through a spatial filter system, formed by a $10 \times$ microscope objective and a $25 \mu \mathrm{m}$ pinhole, the beam is collimated by a lens of $50 \mathrm{~mm}$ focal length. Then, the 2-cm-diameter pulses impinge a $2 \mu \mathrm{m}$ thick pellicle beamsplitter made of a nitrocellulose membrane with a reflectance/transmittance ratio of $45 / 55$ for the bandwidth of 700-900 nm. The transmitted light illuminates the phase modulator. The electrically addressed phase modulator, working in reflection, allows a flexible beam shaping and forms a desired diffraction pattern at the output plane of the dispersion compensation system, formed by a diffractive-refractive lens triplet whose construction details are shown below.

The phase modulator (X8267, Hamamatsu), consists of a PAL-SLM optically addressed by the signal coming from a transmission liquid crystal device (LCD). Thanks to the coupling-optics design, the LCD pixel structure is not transferred into the PAL-SLM. The system performs phase-only modulation provided that the liquid crystal molecular axis of the PAL-SLM is set parallel to the polarization direction of

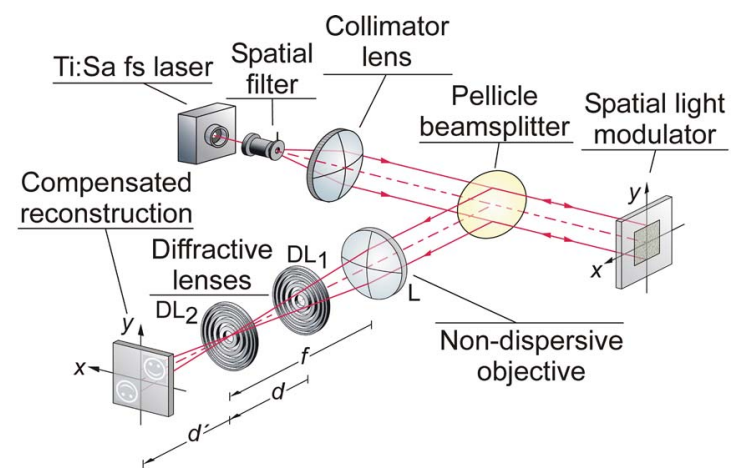

FIG. 1. (Color online) Experimental setup for sub-100-fs real-time holographic reconstruction. 


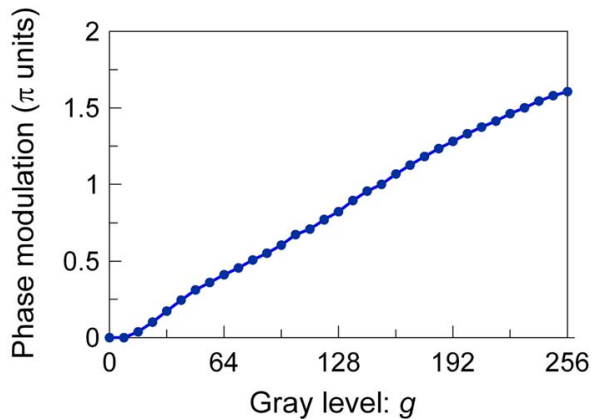

FIG. 2. (Color online) Phase modulation provided by the PAL-SLM for $800 \mathrm{~nm}$.

the femtosecond laser. The response time of the device allows a refreshing rate of a few hertz.

The phase modulator was calibrated for the wavelength of $800 \mathrm{~nm}$ using a polarimetric setup: varying gray-level values displayed in the modulator yield different phase shifts measured as an intensity modulation by means of an analyzer. The angle between the horizontal femtosecond laser polarization and the molecular axis of the PAL-SLM was fixed at $45^{\circ}$. For each gray level, the analyzer was set alternatively at $45^{\circ}$ and $135^{\circ}$ to obtain a normalized intensity modulation. As a result, the integral phase response could be calibrated as a function of the addressing parameter. ${ }^{9}$ The system proved to achieve a phase-only modulation over $3 \pi / 2 \mathrm{rad}$, as shown in Fig. 2. Although the modulation provided by the PAL-SLM depends on wavelength, we have verified that, for our application, the performance of the device for the whole spectrum of our laser can be approximated to the experimental curve in Fig. 2.

The CGH codified in the PAL-SLM is calculated by using an iterative Fourier transform algorithm. Our method is based on the well-known Gerchberg-Saxton algorithm (or error reduction algorithm ${ }^{10}$ but carried out in two stages as proposed by Wyrowski. ${ }^{11}$ The first stage performs iterations by applying only phase freedom, while the second stage restricts softly the phase quantization of the hologram until the desired number of phase levels is reached. As our aim is to reconstruct image holograms, we analyze the convergence of the algorithm by measuring the root mean square (rms) error of the reconstruction with respect to the desired irradiance instead of using efficiency or uniformity criteria. The algorithm comes to the end when the result of the iteration improves the rms error by an amount of $10^{-8}$, compared with the previous one. The time required for calculating a four phase level CGH with $512 \times 512$ pixels, with 23 iterations in the first stage and 38 in the second one, is about $36 \mathrm{~s}$ by using MATLAB running in a personal computer with processor Intel Core 2 Duo T7250 $2.00 \mathrm{GHz}$.

To compensate the diffraction-driven SC, the beam diffracted by the PAL-SLM and reflected by the pellicle beamspliltter is gathered by the highly dispersive setup formed by an achromatic objective $\mathrm{L}$ coupled to a diffractive lens (DL) doublet, $\mathrm{DL}_{1}$ and $\mathrm{DL}_{2}$. At this point it is worth mentioning that the focal distance of a DL is proportional to the frequency of the radiation. The requirements for isotropic SC correction lead to certain constraints regarding the focal lengths and the distances between $\mathrm{L}$ and the DL doublet. ${ }^{6}$ To this end, in our setup, the DLs have an image focal length of $Z_{o}^{\prime}=-Z_{o}=140 \mathrm{~mm}$ for $800 \mathrm{~nm}$, L has a focal length of

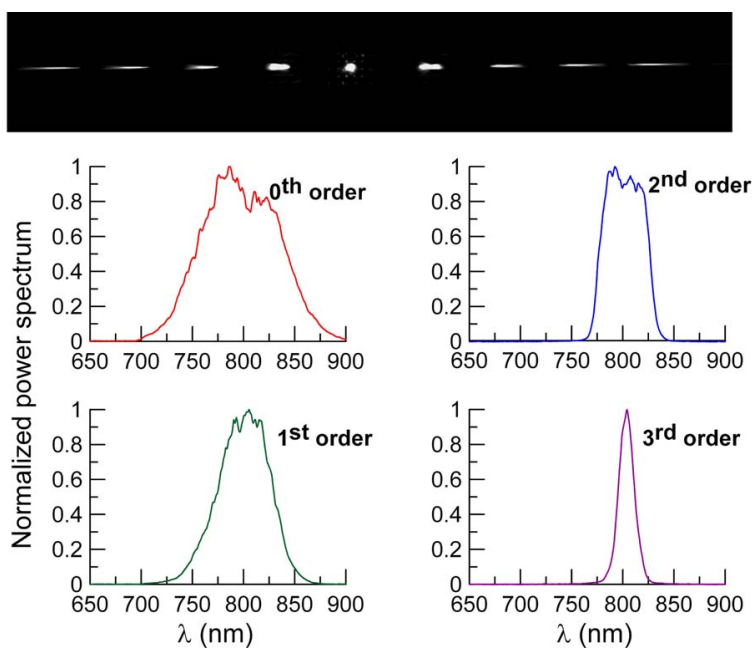

FIG. 3. (Color online) Far field diffraction pattern of a DG recorded in the focal plane of $\mathrm{L}$ (top) and the output spectrum when a pinhole of $5 \mu \mathrm{m}$ is located for different diffraction orders in the spatial position corresponding to the maxima for $800 \mathrm{~nm}$ (bottom).

$f=200 \mathrm{~mm}$, and the axial distances are $d^{\prime}=d=140 \mathrm{~mm}$. See definitions in Fig. 1.

Before discussing experiments demonstrating real-time holographic reconstruction, we first demonstrate the SC compensation, by replacing the PAL-SLM with a simple and well-known diffractive element that introduces angular dispersion, a diffraction grating (DG). In many occasions researchers deliberately use a DG to spatially separate different frequency components of a laser pulse, as in Fouriersynthesis pulse shaping. To this end, a lens is placed one focal length away from the DG and the frequency-to-position mapping, that is, the SC, is found at its focal plane. We employ a Ronchi type DG of 12 lines/mm and the lens L to replicate this experiment. The output image recorded with a charge coupled device (CCD) camera is shown in the upper part of Fig. 3. Each spectral component is focused at a different transversal position as a direct effect of the SC. It results in an elongated spot and a spatial spread of the optical energy at each blurred diffraction maxima. Obviously, this effect should be overcome in other applications requiring high spatiotemporal resolution. With this aim, we use the system in Fig. 1 for reducing the lateral walk-off between the different spectral components at the light spots. The output image recorded with a CCD camera is shown in the upper part of Fig. 4. The improvement in the spatial resolution is noticeable. For instance, for the second-order diffraction maximum, the size of the spot has been reduced approximately by a factor of 10 .

As a second demonstration of the SC correction, the output spectrum of the different diffraction orders has been measured. We place a $5 \mu \mathrm{m}$ radius circular pinhole at the spatial position corresponding to the diffraction order maxima for $800 \mathrm{~nm}$. The output power spectrum of the light passing through the pinhole was measured with a fiber optics spectrometer. The corresponding experimental data are shown in the lower part of Figs. 3 and 4 for the refractive lens system and the SC correction system, respectively. Note that in Fig. 3 the spectral width is reduced as the diffraction order of the DG is increased. The decrease in the available bandwidth at each point is responsible for the pulse broadening. In contrast, the spectrum is almost the same in all the diffraction 

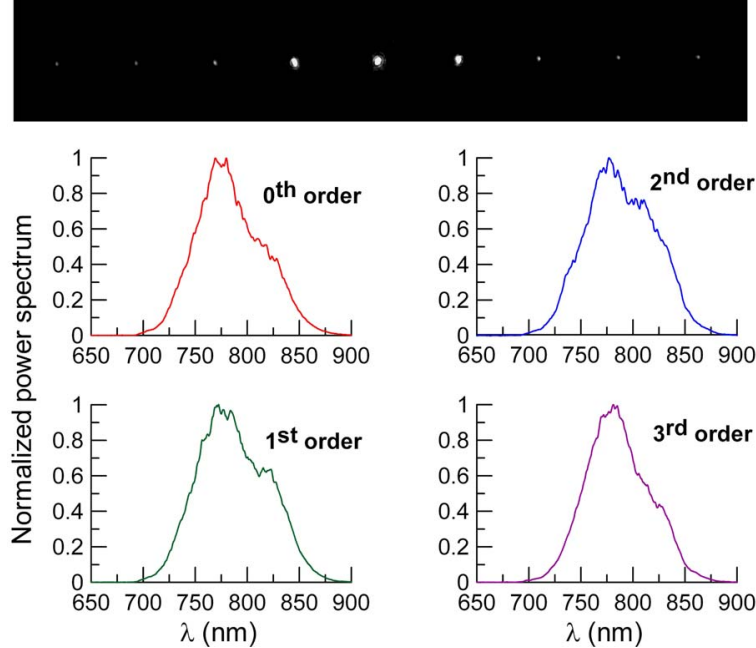

FIG. 4. (Color online) Far field diffraction pattern of a DG recorded in the output plane of the proposed system (top) and the output spectrum when a pinhole of $5 \mu \mathrm{m}$ is located for different diffraction orders in the spatial position corresponding to the maxima for $800 \mathrm{~nm}$ (bottom).

orders in Fig. 4. This corroborates the first-order correction in the SC and, consequently, the pulse broadening due to pulse-front tilt is eliminated. High order distortions would be responsible for the temporal stretching of the pulse. $^{7}$

Next, we show the real-time holographic reconstruction with a femtosecond laser pulse. Figure 5 shows experimental results of one of the CGHs of $512 \times 512$ pixels employed in (a)

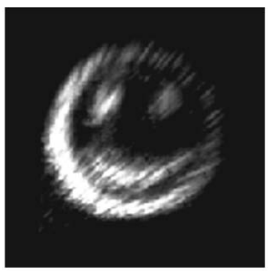

(c)

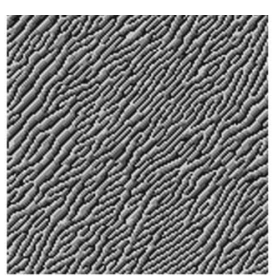

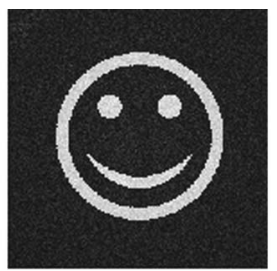

(b)

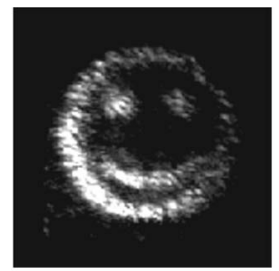

(d)
FIG. 5. (a) Detail of the calculated CGH of a smile picture. (b) Computational reconstruction of the $\mathrm{CGH}$. (c) Optical reconstruction of the $\mathrm{CGH}$ at the focal plane of a refractive lens with the $12 \mathrm{fs}$ laser pulse. (d) Optical reconstruction of the $\mathrm{CGH}$ with the system proposed in Fig. 1. our experience, a smile picture. It has a size of 13.3 $\times 13.3 \mathrm{~mm}^{2}$ in the PAL-SLM and a carrier frequency of 10 lines $/ \mathrm{mm}$. Figures 5(a) and 5(b) show details of the CGH calculated to produce this pattern and the computational reconstruction of the CGH, respectively. The optically reconstructed pattern observed by a CCD image sensor at the focal plane of $\mathrm{L}$ when the DLs are not placed in the setup is shown in Fig. 5(c). This reconstruction provides a blurred image due to the SC caused by the CGH diffraction of the broadband pulsed radiation. After setting the DLs in the setup, the spatial resolution of the output image is increased, see Fig. 5 (d), demonstrating the compensating capabilities of our system. The inferior quality of the optical reconstruction compared to the simulation is mainly due to the residual amplitude modulation introduced by the SLM setup and the speckle pattern produced by the random phase associated with the object in the CGH coding scheme.

In summary, we have experimentally demonstrated the SC compensation of variable Fourier CGH reconstruction with femtosecond pulses, thanks to a dispersion-reversed optical setup. Our first-order SC correction system enables an effective high-resolution sub-100-fs beam shaping.

This research was funded by the Spanish Ministry of Education and Science (under Project No. FIS2007-62217 and Consolider Program No. SAUUL CSD2007-00013) and the Valencian Local Government Department of Trade, Universities and Science (Project No. GV/2007/128). Omel Mendoza-Yero gratefully acknowledges financial support from the UJI-Fundació Caixa Castelló (Bancaixa) Agreement. The authors are very grateful to the Servei Central d'Instrumentació Científica (SCIC) of the University Jaume I for the use of the Ti:sapphire ultrafast laser.

${ }^{1}$ Y. Hayasaki, T. Sugimoto, A. Takita, and N. Nishida, Appl. Phys. Lett. 87, 031101 (2005).

${ }^{2}$ L. Sacconi, E. Froner, R. Antolini, M. R. Taghizadeh, A. Choudhury, and F. S. Pavone, Opt. Lett. 28, 1918 (2003).

${ }^{3}$ D. G. Grier, Nature (London) 424, 810 (2003).

${ }^{4}$ K. Bezuhanov, A. Dreischuh, G. G. Paulus, M. G. Schätzel, and H. Walther, Opt. Lett. 29, 1942 (2004).

${ }^{5}$ S. Nolte, in Ultrafast Lasers: Technology and Applications, edited by M. E. Fermann, A. Galvanauskas, and G. Sucha (Dekker, New York, 2003), Chap. 7.

${ }^{6}$ G. Mínguez-Vega, J. Lancis, J. Caraquitena, V. Torres-Company, and P. Andrés, Opt. Lett. 31, 2631 (2006).

${ }^{7}$ G. Mínguez-Vega, E. Tajahuerce, M. Fernández-Alonso, V. Climent, J. Lancis, J. Caraquitena, and P. Andrés, Opt. Express 15, 278 (2007).

${ }^{8}$ O. E. Martinez, Opt. Commun. 59, 229 (1986).

${ }^{9}$ Y. Igasaki, F. Li, N. Yoshida, H. Toyoda, T. Inoue, N. Mukohzaka, Y. Kobayashi, and T. Hara, Opt. Rev. 6, 339 (1999).

${ }^{10}$ R. W. Gerchberg and W. O. Saxton, Optik (Jena) 35, 237 (1972).

${ }^{11}$ F. Wyrowski, J. Opt. Soc. Am. A 7, 961 (1990). 